\title{
Schemes and the IP-graph
}

\author{
Rachel Camina
}

Received: 22 September 2006 / Accepted: 24 September 2007 / Published online: 12 October 2007 (C) Springer Science+Business Media, LLC 2007

\begin{abstract}
We consider the common-divisor graph of the set of valencies of a naturally valenced scheme, where scheme is defined in the sense of P.-H. Zieschang. We prove structural results about this graph, and thus give restrictions on the set of natural numbers that can occur as the set of valencies of a naturally valenced scheme.
\end{abstract}

Keywords Naturally valenced schemes $\cdot$ IP graph $\cdot$ Kernel $\cdot$ Common-divisor graph

\section{Introduction}

The IP-graph (also known as the common-divisor graph) is defined as follows. Let $G$ be a group acting transitively on the set $\Omega$. The subdegrees of $(G, \Omega)$ are defined to be the cardinalities of the orbits of the action of a point stabilizer $G_{\alpha}$ on $\Omega$. We assume all subdegrees are finite and let $D$ denote the set of subdegrees, these form the vertices of the IP-graph. Two vertices $x$ and $y$ are joined whenever $x$ and $y$ are not coprime. This graph was introduced by Isaacs and Praeger in the early 1990's [3] and generalises a graph introduced by Betram, Herzog and Mann which takes conjugacy class sizes of a finite group as its vertices and, similarly, joins them whenever the sizes are not coprime [1]. We note that P. Neumann has introduced a variant of the IP-graph called the VIP-graph [5].

In 1975 D.G. Higman [2] introduced coherent configurations, these are combinatorial structures that abstract certain features of a group acting on a set. In this paper we aim to extend some of the ideas of [3] and [1] to the setting of coherent configurations. However, unlike Higman, we do not assume our underlying set is finite. Furthermore we use the terminology of P.-H. Zieschang [6] and call our objects schemes. We will give details in the following section, but briefly, a scheme $S$ on $\Omega$

\footnotetext{
R. Camina $(\bowtie)$

Fitzwilliam College, Cambridge CB3 ODG, UK

e-mail: rdc26@dpmms.cam.ac.uk
} 
is a partition of the set $\Omega \times \Omega$, such that $\emptyset \notin S, 1_{\Omega}=\{(\alpha, \alpha): \alpha \in \Omega\} \in S$, for each $s \in S$ we have $s^{*}=\{(\beta, \alpha):(\alpha, \beta) \in s\} \in S$ and finally the regularity condition: given $p, q, r \in S$ there exists a cardinal number $b_{p, q}^{r}$ such that for any $(\alpha, \beta) \in r$ the number of $\gamma \in \Omega$ which satisfy $(\alpha, \gamma) \in p$ and $(\gamma, \beta) \in q$ is given by $b_{p, q}^{r}$. The $b_{p, q}^{r}$ are called the structure constants of the scheme. Let $G$ be a group acting transitively on the set $\Omega$ and extend this action naturally to an action on $\Omega \times \Omega$. The orbits of this action (known as orbitals) give a partition of $\Omega \times \Omega$ which satisfy the conditions above, thus we have the original example of a scheme. In this paper we are concerned with naturally valenced schemes, that is ones in which all structure constants are finite.

Recall that there is a one-to-one correspondence between the orbits of a point stabliser $G_{\alpha}$ on $\Omega$ and the sets $\Delta(\alpha)=\{\beta \in \Omega:(\alpha, \beta) \in \Delta\}$ where $\Delta$ runs over the orbitals of $\Omega \times \Omega$. Thus it is natural to extend the definition of the IP-graph to the setting of naturally valenced schemes. Let $S$ be a naturally valenced scheme on $\Omega$ and fix $\alpha \in \Omega$. For $s \in S$ let $s(\alpha)$ denote the set $\{\beta:(\alpha, \beta) \in s\}$. Then the vertices are given by the set of valencies $\{|s(\alpha)|: s \in S\}$ of the scheme, this set is independent of the choice of $\alpha$ by the regularity condition. Furthermore, for naturally valenced schemes $|s(\alpha)|$ is finite for all $s \in S$. As before, two vertices are joined if they are not coprime. Note that the graph always has a component consisting of the single vertex $\left|1_{\Omega}(\alpha)\right|=1$, we call this the trivial component. In this paper we prove structural results about this graph.

One of the key tools in [1] is the kernel of a subset $A$ of a group $H: \operatorname{ker} A=\{x \in$ $H \mid x A=A\}$. Thus $\operatorname{ker} A$ is the set-stabiliser of $A$ with respect to $H$ acting on $H$ by left multiplication. The kernel is a subgroup of $H$ and since $A$ is a union of $\operatorname{cosets}$ of $\operatorname{ker} A$ it follows that the order of $\operatorname{ker} A$ divides the order of $A$, this fact is used often. In this paper we translate the concept of a kernel and the proofs of [1] to a combinatorial setting to provide results about naturally valenced schemes. We note that for these proofs to work we have to make the additional assumption that $|s(\alpha)|=\left|s^{*}(\alpha)\right|$ for $s \in S$, we say that paired valencies are equal. This holds for a large number of cases. In particular, if the scheme arises from a group $G$ acting transitively on a set $\Omega$, then paired valencies are equal whenever $G$ is a finite group.

Our main result is as follows.

Theorem The IP-graph of a naturally valenced scheme with paired valencies equal, has at most 2 non-trivial connected components. Furthermore,

(a) if the graph has just one non-trivial connected component, that component has diameter $\leq 4$.

(b) if the graph has two non-trivial connected components, one of these is a complete graph and the other has diameter at most 2.

The author would like to thank Peter Cameron, for first mentioning coherent configurations, and the Mathematics Department of the University of Western Australia for their kind hospitality when this work began, in particular John Bamberg, Michael Giudici and Cheryl Praeger for numerous, invaluable, conversations. 


\section{Definitions \& lemmas}

Let $\Omega$ be a set, possibly infinite. For $s$ any subset of $\Omega \times \Omega$ we define its dual subset $s^{*}$ by

$$
s^{*}=\{(\beta, \alpha):(\alpha, \beta) \in s\} .
$$

A subset $s$ is symmetric if $s=s^{*}$. Furthermore, for $\alpha \in \Omega$ we define

$$
s(\alpha)=\{\beta \in \Omega:(\alpha, \beta) \in s\} .
$$

Definition 1 Let $S$ be a partition of $\Omega \times \Omega$. Then $S$ is a naturally valenced scheme on $\Omega$ if the following conditions hold:

(i) $\emptyset \notin S$.

(ii) $1_{\Omega}=\{(\alpha, \alpha): \alpha \in \Omega\} \in S$.

(iii) $s^{*} \in S$ for all $s \in S$.

(iv) If $p, q, r \in S$ and $(\alpha, \beta) \in r$ then

$$
|\{\gamma:(\alpha, \gamma) \in p,(\gamma, \beta) \in q\}|=b_{p, q}^{r} \in \mathbb{N}
$$

and is independent of $(\alpha, \beta)$.

For ease we denote $1_{\Omega}$ simply by 1 . We have the following binary operation on sets $p, q \subseteq \Omega \times \Omega$,

$$
p \circ q:=\{(\alpha, \beta) \mid \exists \gamma \in \Omega \text { such that }(\alpha, \gamma) \in p \&(\gamma, \beta) \in q\}
$$

That this operation corresponds to the complex product defined in [6], is clear from (i) of the following lemma.

Lemma 1 Let $S$ be a naturally valenced scheme on $\Omega, p, q, s \in S$ and $\alpha \in \Omega$.

(i) $p \circ q=\bigcup_{b_{p, q}^{s} \neq 0} s$.

(ii) $|s(\alpha)|$ is finite and independent of $\alpha$.

(iii) $p \circ q$ is a finite union of elements of $S$.

Proof (i) Follows from condition (iv) in definition above.

(ii) We have $|s(\alpha)|=b_{s, s^{*}}^{1}$.

(iii) By (i), $p \circ q$ is a union of elements of $S$. Using (ii), note that $|(p \circ q)(\alpha)|$ is bounded above by $|p(\alpha)||q(\alpha)|$ which is finite.

We denote the structure constant $b_{s, s^{*}}^{1}$ by $k_{s}$ and call $k_{s}$ the valency of $s$. By Lemma 1(ii) $k_{s}$ is a natural number, hence the term 'naturally valenced scheme'. We can now define a graph.

Definition 2 The $I P$-graph of a naturally valenced scheme $S$, denoted by $\mathcal{I P}(S)$, has vertices given by the set of valencies of the scheme, $\left\{k_{s}: s \in S\right\}$. Two vertices, $k_{s}$ and $k_{r}$, are joined if the valencies are not coprime. 
We are often interested in finite subsets of $S$ and the corresponding subset of $\Omega \times \Omega$ they determine. Let $U=\left\{s_{1}, \ldots, s_{n}\right\} \subseteq S$, we denote $\bigcup U \subseteq \Omega \times \Omega$ by $\bar{U}$. Set $k_{U}=$ $\sum_{i=1}^{n} k_{s_{i}}$, then $k_{U}=|\bar{U}(\alpha)|$ for all $\alpha \in \Omega$ and $k_{U}$ is called the valency of $U$.

We make a further hypothesis.

Definition 3 Let $S$ be a scheme. We say that paired valencies are equal if $k_{s}=k_{s^{*}}$ for all $s \in S$.

Suppose $\Omega$ is finite and $S$ is a scheme on $\Omega$. Let $s \in S$ then, $|s|=k_{s}|\Omega|$ and similarly $\left|s^{*}\right|=k_{s^{*}}|\Omega|$. As $|s|=\left|s^{*}\right|$ it follows that our hypothesis is satisfied when $\Omega$ is finite. Our hypothesis is also satisfied when the scheme arises from a finite group $G$ acting transitively on a set $\Omega$.

For completeness a proof of the following lemma is included, alternatively see [6, Lemmas (1.1.4)(i), (1.1.3)(ii) and (1.1.1)(ii)].

Lemma 2 Let $S$ be a naturally valenced scheme on $\Omega$ with paired valencies equal. Suppose $p, q, s \in S$, we have the following identity

$$
k_{s} b_{p, q}^{s}=k_{q} b_{p^{*}, s}^{q}=k_{p} b_{s, q^{*}}^{p}
$$

Proof Fix $\alpha \in \Omega$ and count the number of triangles $(\alpha, \beta, \gamma)$ with $(\alpha, \beta) \in s,(\alpha, \gamma) \in$ $p$ and $(\gamma, \beta) \in q$. This yields $k_{s} b_{p, q}^{s}=k_{p} b_{s, q^{*}}^{p}$. Instead of fixing $\alpha$ we now fix $\gamma$ and again count triangles $(\alpha, \beta, \gamma)$ with $(\alpha, \beta) \in s,(\alpha, \gamma) \in p$ and $(\gamma, \beta) \in q$. This yields $k_{q} b_{p^{*}, s}^{q}=k_{p^{*}} b_{q, s^{*}}^{p^{*}}$. Using our hypothesis we have that $k_{p}=k_{p^{*}}$, and hence $k_{p^{*}} b_{q, s^{*}}^{p^{*}}=k_{p} b_{s, q^{*}}^{p}$. Thus, putting these identities together we have

$$
k_{s} b_{p, q}^{s}=k_{q} b_{p^{*}, s}^{q}=k_{p} b_{s, q^{*}}^{p}
$$

as required.

The first part of the following lemma, that $p \circ q$ is an element of $S$, follows from [6, Lemma (1.5.2)].

Lemma 3 Let $S$ be a naturally valenced scheme on $\Omega$ with paired valencies equal. Suppose $k_{p}$ and $k_{q}$ are coprime. Then $p \circ q$ is an element of $S$. Furthermore $k_{p \circ q}$ divides $k_{p} k_{q}$ and $k_{p \circ q} \geq \max \left\{k_{p}, k_{q}\right\}$.

Proof We apply the identity of the previous lemma to $p, q$ and a third element of $S$ which we denote by $s$. As $k_{p}$ and $k_{q}$ are coprime, the identity $k_{s} b_{p, q}^{s}=k_{q} b_{p^{*}, s}^{q}=$ $k_{p} b_{s, q^{*}}^{p}$ yields that $k_{p} k_{q}$ divides $k_{s} b_{p, q}^{s}$.

By Lemma 1(iii), we know that $p \circ q$ is a finite union of elements of $S$. Suppose $p \circ q=s_{1} \cup \ldots \cup s_{t}$, for some $s_{1}, \ldots, s_{t} \in S$. Fix $\alpha \in \Omega$ and count the number of pairs $(\alpha, \beta) \in p \circ q$ including repetitions. (Consider the tree with root $\alpha$ connected to the $k_{p}$ points in $p(\alpha)$, call these points $\gamma_{r}$ with $1 \leq r \leq k_{p}$. Then each of these $\gamma_{r}$ is connected to the $k_{q}$ points of $q\left(\gamma_{r}\right)$. We count the number of endpoints of this tree.) This gives $k_{p} k_{q}=k_{s_{1}} b_{p, q}^{s_{1}}+\cdots+k_{s_{t}} b_{p, q}^{s_{t}}$. (Alternatively, see [6, Lemma (1.1.3)(iv)].) 
Let $1 \leq l \leq t$ and suppose $b_{p, q}^{s_{l}} \neq 0$. Replacing $s$ with $s_{l}$ in the first paragraph of this proof gives $k_{p} k_{q}$ divides $k_{s_{l}} b_{p, q}^{s_{l}}$. However, from the previous paragraph, we know that $k_{p} k_{q} \geq k_{s_{l}} b_{p, q}^{s_{l}}$. Thus $k_{s_{l}} b_{p, q}^{s_{l}}=k_{p} k_{q}$. So $p \circ q=s_{l}$ and $k_{s_{l}}$ divides $k_{p} k_{q}$.

For ease we denote $p \circ q$ by $l$. We have $k_{l}=|(p \circ q)(\alpha)| \geq|q(\alpha)|=k_{q}$, by Lemma 1(ii). Note that $l^{*}=q^{*} \circ p^{*}$, and thus $k_{l^{*}} \geq k_{p}$. However, by our hypothesis $k_{l^{*}}=k_{l}$ and our proof is complete.

We let $d\left(k_{p}, k_{q}\right)$ denote the distance between two vertices in the IP-graph. The following lemma follows from [6, Lemma (1.4.4)], we include the proof for completeness.

Lemma 4 Let $S$ be a naturally valenced scheme on $\Omega$ with paired valencies equal and let $p$ and $q$ be elements in $S$ with $k_{q}>k_{p}$ and $d\left(k_{p}, k_{q}\right) \geq 3$. Then there exists an element $s$ in $S$ such that $s=p \circ q, k_{s}=k_{q}$ and $p^{*} \circ p \circ q=q$.

Proof Using the previous lemma and that the distance between $k_{p}$ and $k_{q}$ is at least 3 , gives that $s$ is an element of $S$ and $k_{s}=k_{q}$. We now repeat the argument using the elements $p^{*}$ and $s$. Note that $k_{p^{*}}=k_{p}$ by assumption. Thus, $p^{*} \circ s$ is an element of $S$ and $k_{p^{*} \circ s}=k_{s}=k_{q}$. Furthermore, since $1 \subseteq p^{*} \circ p$ it follows that $q \subseteq p^{*} \circ p \circ q=$ $p^{*} \circ s$. So, $q=p^{*} \circ p \circ q$.

The previous lemma motivates the following definition.

Definition 4 Let $s \subseteq \Omega \times \Omega$. We define the kernel of $s$ as follows:

$$
\operatorname{ker} s=\{(\alpha, \beta) \in \Omega \times \Omega \mid\{(\alpha, \beta)\} \circ s \subseteq s\} .
$$

Lemma 5 Let $s \subseteq \Omega \times \Omega$. Then

(i) $1_{\Omega} \subseteq$ kers.

(ii) $(\alpha, \beta) \in$ kers iff $s(\beta) \subseteq s(\alpha)$.

(iii) Let $S$ be a naturally valenced scheme on $\Omega$ and $U$ be a finite subset of $S$. Then

$$
(\alpha, \beta) \in \operatorname{ker} \bar{U} \text { iff } \bar{U}(\alpha)=\bar{U}(\beta) .
$$

Moreover, $\operatorname{ker} \bar{U} \circ \bar{U}=\bar{U}$.

Proof (i) is clear.

(ii) $(\alpha, \beta) \in \operatorname{ker} s$ iff $(\beta, \gamma) \in s \Rightarrow(\alpha, \gamma) \in s$ iff $s(\beta) \subseteq s(\alpha)$.

(iii) As $U$ is a finite subset of $S$ it follows that $k_{U}=|\bar{U}(\alpha)|=|\bar{U}(\beta)|$ for all $\alpha, \beta \in \Omega$, by Lemma 1(ii). Applying this to (ii) gives $(\alpha, \beta) \in \operatorname{ker} \bar{U}$ iff $\bar{U}(\alpha)=\bar{U}(\beta)$.

Finally, note that the definition of $\operatorname{ker} \bar{U}$ implies that $\operatorname{ker} \bar{U} \circ \bar{U} \subseteq \bar{U}$. Equality follows from (i).

Lemma 5(iii) motivates us to define the following equivalence relations. 
Definition 5 Let $S$ be a naturally valenced scheme on $\Omega$ and $U$ a finite subset of $S$. We define the equivalence relation $R_{U}$ as follows:

$$
\alpha R_{U} \beta \text { iff } \bar{U}(\alpha)=\bar{U}(\beta) .
$$

We denote the equivalence class containing $\alpha$ by $[\alpha]_{U}$.

Lemma 6 Let $S$ be a naturally valenced scheme on $\Omega$ with paired valencies equal. Let $U$ be a finite subset of $S$ and $R_{U}$ the equivalence relation defined above.

(i) Then $\operatorname{ker} \bar{U}=\bigcup_{\alpha \in \Omega}\left([\alpha]_{U} \times[\alpha]_{U}\right)$. Furthermore, $\operatorname{ker} \bar{U}$ is a symmetric subset of $\Omega \times \Omega$.

(ii) $|\operatorname{ker} \bar{U}(\alpha)| \leq k_{U}$

(iii) Let $s \in S$, then kers is a finite union of elements of $S$. Furthermore, $|\operatorname{ker} s(\alpha)|=$ $\left|[\alpha]_{s}\right|$ divides $k_{s}$.

(iv) Suppose $1 \neq s \in S$ then $s \cap \operatorname{ker} s=\emptyset$.

Proof (i) The first part follows from Lemma 5(iii). That $\operatorname{ker} \bar{U}$ is symmetric is now clear.

(ii) Let $\gamma \in \bar{U}(\alpha)$. Then $\operatorname{ker} \bar{U}(\alpha) \subseteq \bar{U}^{*}(\gamma)$ by (i). Since paired valencies are equal, $\left|\bar{U}^{*}(\gamma)\right|=|\bar{U}(\gamma)|$ and by Lemma 1 (ii) $|\bar{U}(\gamma)|=|\bar{U}(\alpha)|=k_{U}$, hence result.

(iii) Let $r, s \in S$. Note that, $(\alpha, \beta) \in \operatorname{ker} s \cap r$ iff $s(\alpha)=s(\beta)$ and $(\alpha, \beta) \in r$ iff $b_{s, s^{*}}^{r}=b_{s, s^{*}}^{1}=k_{s}$. This condition is independent of the choice of $(\alpha, \beta)$, thus kers is a union of elements of $S$, and by (ii) kers is a finite union of elements of $S$. Furthermore, let $\gamma \in s(\alpha)$. Then $\operatorname{ker} s(\alpha)=[\alpha]_{s} \subseteq s^{*}(\gamma)$ by (i). Thus $\operatorname{ker} s(\alpha)$ is a finite union of equivalence classes $\left[\alpha_{i}\right]_{s}$ for $1 \leq i \leq n$, say. Now, as kers is a finite union of elements of $S$, it follows that $|\operatorname{ker} s(\alpha)|$ is independent of $\alpha$. Thus, $\left|[\alpha]_{s}\right|=|\operatorname{ker} s(\alpha)|$ divides $\left|s^{*}(\gamma)\right|=k_{s}$, as required.

(iv) Suppose $(\alpha, \beta) \in \operatorname{ker} \Omega \cap$. Since kers is symmetric by (i) we have $\{(\beta, \beta)\}=$ $\{(\beta, \alpha)\} \circ\{(\alpha, \beta)\} \subset$ kers $\circ s=s$, a contradiction as $s$ was assumed to be nontrivial.

Definition 6 Suppose $s \subseteq \Omega \times \Omega$.

(i) We say that $s$ is o-closed if $s$ is symmetric and $s \circ s \subseteq s$.

(ii) We define the o-closure of $s$, denoted by $\langle s\rangle$, to be the smallest o-closed subset containing $s$.

Remark (i) Let $U$ be a finite subset of $S$ and suppose $\bar{U}$ is o-closed. Then $1 \in U$.

(ii) Suppose $s \subseteq \Omega \times \Omega$ is symmetric, then $\langle s\rangle=\bigcup_{n \in \mathbb{N}_{\geq 1}} \underbrace{s \circ \cdots \circ s}_{n}$.

(iii) Let $s \in S$ then kers is o-closed by Lemma 6(i).

Lemma 7 Let $S$ be a naturally valenced scheme on $\Omega$. Let $U$ and $V$ be finite subsets of $S$. Furthermore, suppose $\bar{V}$ is o-closed. Then the following defines an equivalence relation $R_{U, V}$ on $\Omega$ :

$$
\alpha R_{U, V} \beta \text { iff } \bar{U}(\alpha)=\bar{U}(\beta) \&(\alpha, \beta) \in \bar{V} .
$$

Moreover $R_{U, V}$ is a refinement of the relation $R_{U}$. 
Proof First note that the relation $S_{V}$ defined by $\alpha S_{V} \beta$ iff $(\alpha, \beta) \in \bar{V}$ is an equivalence relation. This follows from the properties of $V$, namely: reflexivity follows since $1 \in V, S_{V}$ is symmetric since $\bar{V}$ is, and transitivity follows from o-closure.

That $R_{U, V}=R_{U} \cap S_{V}$ is an equivalence relation refining $R_{U}$, is now clear.

Lemma 8 Let $S$ be a naturally valenced scheme on $\Omega$ with paired valencies equal and $\alpha \in \Omega$.

(i) Let $s \in S$ and $U$ be a finite subset of $S$. Suppose $\bar{U}$ is o-closed and is contained in kers. Then $k_{U}$ divides $k_{s}$.

(ii) Let $p, q \in S$ satisfy $p^{*} \circ p \subseteq \operatorname{ker} q$. Then there exists a finite subset $U$ of $S$ such that $\bar{U}=\left\langle p^{*} \circ p\right\rangle$. Furthermore, $k_{U}$ divides $k_{q}$.

Proof (i) Let $\gamma \in s(\alpha)$ and $[\alpha]_{s, U}$ denote the equivalence class containing $\alpha$ under $R_{s, U}$. Then $[\alpha]_{s, U} \subseteq s^{*}(\gamma)$, by Lemmas 6(i) and 7. It follows that $s^{*}(\gamma)$ can be written as a union of equivalence classes with respect to the relation $R_{s, U}$. Furthermore, as $\bar{U} \subseteq$ kers it follows that $\bar{U}(\alpha)=[\alpha]_{s, U}$. Finally, as $U$ is a finite subset of $S$ it follows by Lemma 1 (ii), that $|\bar{U}(\alpha)|=k_{U}$ is independent of $\alpha$, and the result follows since $\left|s^{*}(\gamma)\right|=k_{s}$.

(ii) Note that, $p^{*} \circ p \subseteq \operatorname{ker} q$ implies that $\left\langle p^{*} \circ p\right\rangle \subseteq \operatorname{ker} q$ since $\operatorname{ker} q$ is o-closed by Lemma 6(i). By Lemma 1(iii), we know that $p^{*} \circ p$ is a finite union of elements of $S$, and thus, by the definition of o-closure, $\left\langle p^{*} \circ p\right\rangle$ is a union of elements of $S$. Thus, there exists a subset $U$ of $S$ such that $\left\langle p^{*} \circ p\right\rangle=\bar{U}$. That $U$ is a finite subset of $S$ follows from Lemma 6(iii), which says that ker $q$ is a finite union of elements of $S$. We can now apply (i).

\section{Results}

Let $S$ be a naturally valenced scheme on $\Omega$ with paired valencies equal. Denote the IP-graph associated to $S$ by $\mathcal{I} \mathcal{P}$. In this section we prove structural results about $\mathcal{I} \mathcal{P}$. The results can be interpreted as restrictions on the set of natural numbers which can occur as the set of valencies of $S$.

The proofs in this section mimic the proofs of [1]. In this way we provide alternative proofs to Theorems A and C of [3] and Theorem E of [4] for the restricted case when paired valencies are equal.

Note that $\mathcal{I P}$ always has a component consisting of the single vertex $\left|1_{\Omega}(\alpha)\right|=1$, we call this the trivial component. The following theorem considers the number of non-trivial components of $\mathcal{I} \mathcal{P}$.

Theorem 1 Let $S$ be a naturally valenced scheme on $\Omega$ with paired valencies equal. Then the number of non-trivial components of $\mathcal{I P}$ is $\leq 2$.

Proof Suppose otherwise and let $s, q, p \in S$ lie in different components of $\mathcal{I P}$ with $k_{s}>k_{q}>k_{p}>1$. Then $p^{*} \circ p \circ q=q$ and $p^{*} \circ p \circ s=s$, by Lemma 4. Now apply Lemma 8(ii) to yield $\left|\left\langle p^{*} \circ p\right\rangle(\alpha)\right|$ divides $\left(k_{s}, k_{q}\right)=1$. This gives us a contradiction as $p$ was chosen to be non-trivial. 
Theorem 2 Let $S$ be a naturally valenced scheme on $\Omega$ with paired valencies equal. Then the diameter of $\mathcal{I} \mathcal{P}$ is at most 4.

Proof Suppose otherwise, let $1 \neq p, q \in S$ satisfy $d\left(k_{p}, k_{q}\right)=5$ in $\mathcal{I P}$ and $k_{p}<k_{q}$. Let $s \in S$ be such that

$$
d\left(k_{p}, k_{s}\right)=3 \text { and } d\left(k_{q}, k_{s}\right)=2 .
$$

If $k_{p}<k_{s}$ then $p^{*} \circ p$ lies in the kernel of both $s$ and $q$ by Lemma 4. But this yields a contradiction, since $\left|\left\langle p^{*} \circ p\right\rangle(\alpha)\right|>1$ but by Lemma 8(ii) divides both $k_{s}$ and $k_{q}$, contradicting $d\left(k_{s}, k_{q}\right)=2$. Thus $k_{s}<k_{p}<k_{q}$. Recall that paired valencies are equal so $k_{p}=k_{p^{*}}$. Now apply Lemma 4 to the pair $s, p$ and the pair $p^{*}, q$. Thus $s^{*} \circ s \circ p=p$ and $p \circ p^{*} \circ q=q$. Combining these gives

$$
\begin{aligned}
s^{*} \circ s \circ q & =s^{*} \circ s \circ\left(p \circ p^{*} \circ q\right) \\
& =\left(s^{*} \circ s \circ p\right) \circ p^{*} \circ q \\
& =p \circ p^{*} \circ q \\
& =q .
\end{aligned}
$$

Thus, by Lemma 8(ii), we have that $\left|\left\langle s^{*} \circ s\right\rangle(\alpha)\right|$ is a non-trivial, common divisor of both $k_{q}$ and $k_{p}$, a contradiction.

We do not know if this bound is sharp in the sense that we know of no scheme with IP-graph of diameter 4. Examples of schemes with IP-graphs of diameter 3 can be found in [3].

Lemma 9 Let $S$ be a naturally valenced scheme on $\Omega$ with paired valencies equal. Suppose there exists $p, q_{1}, q_{2} \in S$ such that $k_{p}, k_{q_{1}}$ and $k_{q_{2}}$ are pairwise coprime and $1<k_{p}<k_{q_{1}}<k_{q_{2}}$. Then there exists an $i$ such that $d\left(k_{p}, k_{q_{i}}\right)=2$ and furthermore there exists $s \in S$ such that $k_{s}>k_{q_{i}}$ and $k_{s}$ divides $k_{p} k_{q_{i}}$.

Proof If the conditions of the lemma are not satisfied then $p^{*} \circ p \subseteq \operatorname{ker} q_{j}$ for $j=1,2$ by Lemma 3 and the proof of Lemma 4 . But $\left(k_{q_{1}}, k_{q_{2}}\right)=1$, yielding a contradiction by Lemma 8(ii).

The above lemma restricts which graphs can appear as IP-graphs. In particular it implies that there is much connectivity in these graphs. For example the following three graphs cannot appear as IP-graphs, or components of IP-graphs by Lemma 9.
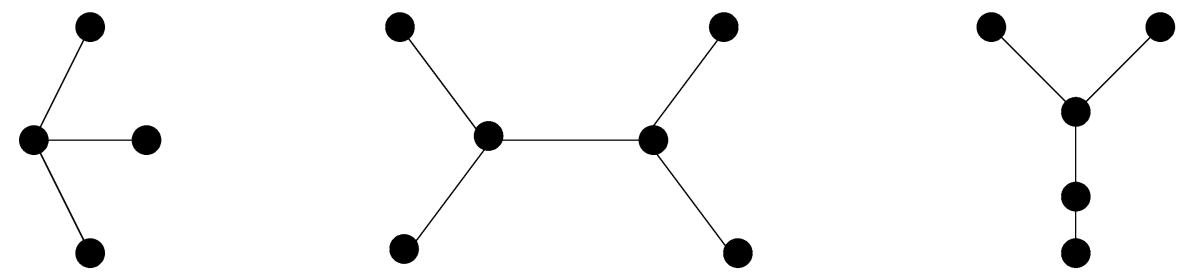
In particular, in the following corollary we prove that stars cannot appear as IPgraphs. Recall, the degree of a vertex of a graph is the number of edges incident to that vertex.

Corollary 1 Let $S$ be a naturally valenced scheme on $\Omega$ with paired valencies equal. Let $\mathcal{D}$ be a non-trivial component of $\mathcal{I} \mathcal{P}$. Suppose $\mathcal{D}$ has a central vertex (i.e. a vertex connected to all other vertices) and furthermore suppose $\mathcal{D}$ has at least 4 vertices. Then $\mathcal{D}$ has at most 2 vertices of degree 1.

Proof Suppose $\mathcal{D}$ has three vertices of valency 1 , call them $k_{p}, k_{q_{1}}$ and $k_{q_{2}}$. These three vertices $k_{p}, k_{q_{1}}$ and $k_{q_{2}}$, are connected to the central vertex, thus $k_{p}, k_{q_{1}}$ and $k_{q_{2}}$ are pairwise coprime. We can now apply Lemma 9, thus we have a vertex $k_{s}$ which divides $k_{p} k_{q_{i}}$. Note $k_{s}$ cannot be the central vertex, as it is coprime to $k_{q_{j}}$ where $j \neq i$. However, $k_{s}$ is connected to both $k_{p}$ and $k_{q_{i}}$, contradicting that these vertices are of degree 1 .

Similar techniques also prove the following.

Proposition 1 Suppose $S$ is a naturally valenced scheme on $\Omega$ with paired valencies equal. Suppose $\mathcal{I} \mathcal{P}$ is disconnected with connected non-trivial components $\mathcal{D}_{1}$ and $\mathcal{D}_{2}$. Suppose the minimum valency (greater than 1 ) lies in $\mathcal{D}_{1}$. Then $\mathcal{D}_{2}$ is complete and $\mathcal{D}_{1}$ has a central vertex, namely the maximal valency of $\mathcal{D}_{1}$.

Proof Suppose $\mathcal{D}_{2}$ is not complete. Let $k_{p}$ be the minimal valency, with $k_{p}>1$. By assumption $k_{p}$ lies in $\mathcal{D}_{1}$. Choose two coprime valencies in $\mathcal{D}_{2}$ and label them $k_{q_{1}}$ and $k_{q_{2}}$. Now apply Lemma 9 to get a contradiction.

We show that the maximal valency in $\mathcal{D}_{1}$ is central. Suppose not and choose a valency of $\mathcal{D}_{1}$ coprime to the maximal valency of $\mathcal{D}_{1}$. Furthermore choose a valency of $\mathcal{D}_{2}$. We label these three vertices as $k_{p}, k_{q_{1}}$ and $k_{q_{2}}$, where $k_{p}<k_{q_{1}}<k_{q_{2}}$. Note that the maximal valency of $\mathcal{D}_{1}$ is $k_{q_{j}}$ for $j=1$ or 2 . Also note that the three valencies are pairwise coprime. Thus we can apply Lemma 9 and we have a $k_{q_{i}}$ and a $k_{s}$. If $k_{q_{i}} \in \mathcal{D}_{2}$ then $k_{p}$ and $k_{q_{i}}$ lie in different components and no $k_{s}$ can exist. If $k_{q_{i}} \in \mathcal{D}_{1}$, either $k_{q_{i}}$ is the maximal valency of $\mathcal{D}_{1}$ in which case $k_{s}$ does not exist, or $k_{q_{i}}$ is not the maximal valency of $\mathcal{D}_{1}$ and then $k_{p}$ is in $\mathcal{D}_{2}$ and, again, $k_{s}$ does not exist.

\section{References}

1. Bertram, E.A., Herzog, M., Mann, A.: On a graph related to conjugacy classes of groups. Bull. Lond. Math. Soc. 22, 569-573 (1990)

2. Higman, D.G.: Coherent configurations. Geom. Ded. 4, 1-32 (1975)

3. Isaacs, I.M., Praeger, C.E.: Permutation group subdegrees and the common divisor graph. J. Algebra 159, 158-175 (1993)

4. Kaplan, G.: On groups admitting a disconnected common divisor graph. J. Algebra 193, 616-628 (1997)

5. Neumann, P.M.: Coprime suborbits of transitive permutation groups. J. Lond. Math. Soc. (2) 47(2), 285-293 (1993)

6. Zieschang, P.-H.: Theory of Association Schemes. Springer Monographs in Mathematics. Springer, Berlin (2005) 